\title{
NATURAL FAMILIES OF TRAJECTORIES: CONSERVATIVE
}

\section{FIELDS OF FORCE*}

\author{
BY
}

\author{
EDWARD KASNER
}

\section{INTRODUCTION.}

The families of curves considered in this paper may be defined as the extremals connected with variation problems of the form

$$
\int F d s=\text { minimum, }
$$

where $F$ is any point function and $d s$ is the element of are in the space considered. $\dagger$ In the plane such a family contains $\propto^{2}$ curves; in ordinary space, the number is $\infty^{4}$; and in the general case of $n$ dimensions the number is $\infty^{2(n-1)}$, one passing through every point in every direction. The results obtained hold for the general case, though for simplicity they will be written out in the following only for three dimensions.

Any system of curves connected with an integral (1) we shall refer to as a natural system or family. The name seems justified in view of the many important geometrical and physical investigations which lead to variation problems of type (1). The following six are perhaps the most noteworthy.

1) Trajectories in a conservative field of force. Let a unit particle move in a field due to the work function (negative potential) $W(x, y, z)$. The totality of motions, for all initial conditions, given by the equations

$$
\ddot{x}=W_{x}, \quad \ddot{y}=W_{y}, \quad \ddot{z}=W_{,},
$$

leads to $\infty^{5}$ trajectories. These may be grouped according to the values of $h$, the constant of energy defined by the energy equation

$$
v^{2}=\dot{x}^{2}+\dot{y}^{2}+\dot{z}^{2}=2(W+h) .
$$

For each value of $h$ we obtain a family of $\infty^{4}$ trajectories. Such a family is a

* Presented to the Society April 28, 1905 and Ootober 30, 1906, in two papers entitled Velocity systems in the dymamics of a particle and The motion of a particle under conservative forces.

t The space is supposed to be euclidean of any dimensionality. The results admit of direct extension to all spaces of constant curvature. Geodesic circles then play the rôle of ordinary circles. 
natural family, since by the principle of least action we have

$$
\int \sqrt{W+h} d s=\text { minimum. }
$$

The complete system * of $\infty^{5}$ trajectories in a conservative field is thus composed of $\infty^{1}$ natural families.

2) Brachistochrones. In the case of conservative forces the brachistochrone problem leads to the integral

$$
\int d t=\int \frac{d s}{\sqrt{W+h}}
$$

The complete system of brachistochrones is thus made up of $\infty^{1}$ natural families.

3) Catenaries. When a homogeneous flexible inextensible string is acted on by conservative forces, the forms of equilibrium (catenaries in the general sense) are determined by rendering

$$
\int(W+h) d s
$$

a minimum. Hence here also we have $\infty^{1}$ natural families, one for each value of $h \cdot \dagger$

4) Optics. Consider an isotropic medium in which the index of refraction $\nu$ varies from point to point. The paths of light in such a medium, according to Fourier's principle, are determined by minimizing the integral

$$
\int \nu d s,
$$

and hence form a single natural family.

5) Geodesics. On a surface whose squared element of length (first fundamental form) is

$$
\lambda(x, y)\left(d x^{2}+d y^{2}\right),
$$

the determination of the geodesics leads to the minimizing of

$$
\int \sqrt{\lambda} d s
$$

hence the representing curves in the $x, y$ plane form a natural family. Thus if any surface is represented conformally on a plane the geodesics are pictured by a natural family of curves in that plane. The extension to more variables is obvious : any natural family in any space may be obtained by conformal representation from the geodesics of some variety in higher space.

* Such complete systems, for both conservative and non-conservatjue forces, were treated in the author's earlier papers in these Transactions, vol. 7 (1906), pp. 401-424, and vol. 8 (1907), pp. 135-158.

† The total systems of $\infty^{5}$ catenaries and $\infty^{5}$ brachistochrones have geometric properties distinct from each other and from those of the $\infty^{5}$ trajectories. This subject will be treated in another paper. 
6) Contact transformations. LiE* has indicated the importance of that category of infinitesimal contact transformation, whose characteristic function is, in the case of the plane, of the particular form

$$
\Omega(x, y) \sqrt{1+y^{\prime 2}} .
$$

These are characterized by the fact that the elements at each point are converted into the elements of a circle about that point as center. The path curves of such a transformation form a natural family.

The principal object of this paper is to obtain a complete geometric characterization of natural families of curves. It is shown that two properties, referred to as $A$ and $B$, are necessary and sufficient. The first states that if the osculating circles of those curves of the family which pass through a given point $p$ are constructed at that point, they will have a second point $P$ in common; and thus (in the case of three dimensions) form a bundle. It is shown as a consequence of $A$ that three (or $n$ in the case of $n$ dimensions) of the circles in such a bundle will have four-point contact with the corresponding curves. Property $B$ states that these hyperosculating circles will be mutually orthogonal.

The next result is a reciprocity principle for natural systems. With each natural system $S$ there is associated a second natural system $S^{\prime}$ as follows: the bundle of osculating circles constructed at a point $p$ for the $S$ curves is at the same time the bundle of osculating circles at the second point $\boldsymbol{P}$ of the bundle for the $S^{\prime}$ curves. The relation is of course mutual.

The correspondence of $p$ and $P$ leads to a point transformation whose character may be described in terms of a known category of space deformations referred to as of the DaRBoux type. The result is an equivalent for property $B$.

The class of systems having property $A$ is of course far more extensive than the class of natural systems. All such systems are here termed velocity systems. They arise in fact in the study of certain curves (velocity curves) which are definitely related to the motion of a particle in an arbitrary field of force. For a given value of the speed, $v=v_{0}$, there are $\infty^{4}$ of these curves; they are defined by the fact that if a particle is projected in the direction of one of them with speed $v_{0}$ the trajectory described will osculate the velocity curve.

The last part of the paper is devoted to the two-dimensional theory. Here the velocity type includes not only the natural type but also the isogonal type (formed by all the $\infty^{2}$ isogonals of a given set of $\infty^{1}$ curves). The comparison

* Die infinilesimalen Berührungstransformationen der Mechanik, Lei pziger Berich te (1889), pp. 145-153, and Lie-SchefFers, Geometrie der Beriihrungstransformationen, p. 102. A more complete treatment is given by Vessiot, Bulletin de la Societe Mathematiquede France, vol. 34 (1906), pp. 230-269. The present writer has shown that the alternant of any two of these contact transformations is a point transformation. See Bulletin of the American Mathematical Society, vol. 14 (1908), p. 356.

Trans. Am. Math. Soc. 14 
of these two types leads to simple results. A transformation of curvature elements is found which interchanges the types. ScHeFfer's theory of isogonal trajectories * is supplemented with respect to an essential feature.

\section{§1. Osculating Circles-Property $A$.}

The extremals connected with an integral of form (1), which in the case of three dimensions we write

$$
\int F(x, y, z) v^{\prime} \overline{1+y^{\prime 2}+z^{\prime 2}} d x,
$$

are given by the EuLER equations $\dagger$

where

$$
\begin{aligned}
& \dot{y}^{\prime \prime}=\left(L_{y}-y^{\prime} L_{x}\right)\left(1+y^{\prime 2}+z^{\prime 2}\right), \\
& z^{\prime \prime}=\left(L_{z}-z^{\prime} L_{x}\right)\left(1+y^{\prime 2}+z^{\prime 2}\right),
\end{aligned}
$$

Thus any natural family of curves is represented by differential equations of the form (2), where $L$ is an arbitrary point function.

Of the $\infty^{4}$ curves in such a family, $\infty^{2}$ pass through any given point, one in each direction. Our first result is

Theorem 1. The $\infty$ murves of any natural family have this property: the circles which osculate at any point $p$ of space the various curves passing through that point have a second point $P$ in common, and thus form a bundle.

This property we shall refer to as property $A$. In the discussion it will be convenient to decompose it into these two statements, which also relate to the $\infty^{2}$ curves through a given point :

$\left(A_{1}\right)$ The osculating planes constructed at the common point form a pencil.

$\left(A_{2}\right)$ The centers of curvature lie in a plane perpendicular to the axis of the pencil of osculating planes.

A synthetic proof of the theorem stated is easily obtained from the dynamical interpretation 1) of the introduction. Property $A_{1}$ results from the fact that the osculating plane of a trajectory always passes through the force vector. Property $A_{2}$ is proved by noting that the trajectories through a given point which correspond to the same value of the total energy $h$ are all described with the same initial velocity $v_{0}$. The radius of curvature at the initial point is

$$
r=v_{0}^{2} / N \text {, }
$$

where $N$ denotes the component of the force along the principal normal. Since $N$ is the orthogonal projection of a fixed vector, the locus of its terminal point

* Leipziger Berichte, 1898 and 1900 ; Mathematische Annalen, vol. 60 (1905).

$\dagger$ Throughout the paper primes refer to total derivatives with respect to $x$; and literal subscripts to partial derivatives. 
will be a sphere through the initial point. The conclusion then follows from the fact that $r$ varies inversely as $N$.

'The following analytical discussion has the advantage of answering the converse question which naturally arises: Are there other systems with property $A$ ?

The differential equations of any system of $\infty^{4}$ space curves, one determined by each lineal element of space, may be assumed in the form

$$
y^{\prime \prime}=f\left(x, y, z, y^{\prime}, z^{\prime}\right), \quad z^{\prime \prime}=g\left(x, y, z, y^{\prime}, z^{\prime}\right) .
$$

Property $A_{1}$ requires that at each point there shall be a certain direction through which all the osculating planes at that point must pass. Let the direction in question be given by the ratios of three arbitrary point functions

$$
\phi(x, y, z), \quad \psi(x, y, z), \quad \chi(x, y, z) ;
$$

then the requisite condition is

$$
\frac{z^{\prime \prime}}{\bar{y}^{\prime \prime}}=\frac{\chi-z^{\prime} \phi}{\psi-y^{\prime} \phi} .
$$

Property $A_{2}$ requires that the center of curvature shall lie in a plane perpendicular to the direction (4); hence

$$
\phi X+\psi Y+\chi Z=1,
$$

where $X, Y, Z$ denote the coördinates of the center relative to axes with the common point as origin. Using the general formulas for the center of curvature, and combining with (5), we find

Theorem 2. The differential equations of any system of curves possessing property $A$ are of the form

$$
\begin{aligned}
& y^{\prime \prime}=\left(\psi-y^{\prime} \phi\right)\left(1+y^{\prime 2}+z^{\prime 2}\right), \\
& z^{\prime}=\left(\chi-z^{\prime} \phi\right)\left(1+y^{\prime 2}+z^{\prime 2}\right),
\end{aligned}
$$

where $\phi, \psi, \chi$ are arbitrary functions of $x, y, z$. The converse is valid also.

The equations (2) are seen to be included in this form, hence the result certainly holds for our natural systems, as stated in theorem 1 .

\section{§ 2. Hyperosculations-Property $B$.}

The circles of curvature at a given point, for any system of the form (7), constitute a bundle. We now inquire whether any of these circles correspond to four-point, instead of three-point, contact.

If a twisted curve is to have an hyperosculating circle of curvature at a given point two conditions must be satisfied, namely,

$$
\left|\begin{array}{lll}
1 & y^{\prime} & z^{\prime} \\
0 & y^{\prime \prime} & z^{\prime \prime} \\
0 & y^{\prime \prime \prime} & z^{\prime \prime \prime}
\end{array}\right|=0,
$$




$$
\frac{d r}{d s}=0
$$

The first of these states that the osculating plane has four-point contact with the curve; the second, in which $r$ denotes the radius of curvature, is the condition for the existence of an osculating helix, i. e., one with four-point contact. When both conditions hold the helix is simply the circle of curvature which then has hyper-contact.

Applying these conditions to the curves defined by (7), we find, from (8),

$$
\left(\psi-y^{\prime} \phi\right) \chi^{\prime}-\left(\chi-z^{\prime} \phi\right) \psi^{\prime}+\left(y^{\prime} \chi-z^{\prime} \psi\right) \phi^{\prime}=0 ;
$$

and,* from (9),

$$
\left(1+y^{\prime 2}+z^{\prime 2}\right) \Sigma \phi \phi^{\prime}-\left(\phi+y^{\prime} \psi+z^{\prime} \chi\right)\left\{\begin{array}{c}
\left(1+y^{2}+z^{\prime 2}\right) \Sigma \phi^{2}+\phi^{\prime}+y^{\prime} \psi^{\prime}+z^{\prime} \chi^{\prime} \\
-\left(\phi+y^{\prime} \psi+z^{\prime} \chi\right)^{2}
\end{array}\right\}=0,
$$

where the indicated summations extend over $\phi, \psi, \chi$ and where $\phi^{\prime}$, for example, denotes $\phi_{x}+y^{\prime} \phi_{y}+z^{\prime} \phi_{z}$.

Since we wish to discuss the $\infty^{2}$ curves through a given point, we may simplify our equations considerably by taking the axis of abscissas in the special direction (4). Then, at the selected point, $\psi$ and $\chi$ vanish, and the above equations reduce to

$$
\begin{gathered}
y^{\prime} \chi^{\prime}-z^{\prime} \psi^{\prime}=0, \\
\left(y^{\prime 2}+z^{\prime 2}\right)\left(\phi^{\prime}-\phi^{2}\right)-\left(y^{\prime} \psi^{\prime}+z^{\prime} \chi^{\prime}\right)=0 .
\end{gathered}
$$

Neglecting the trivial solutions for which $y^{\prime 2}+{z^{\prime 2}}^{2}$ vanishes, we may reduce this pair of simultaneous equations to the form

$$
\frac{\Psi_{x}+y^{\prime} \psi_{y}+z^{\prime} \psi_{x}}{y^{\prime}}=\frac{\chi_{x}+y^{\prime} \chi_{y}+z^{\prime} \chi_{z}}{z^{\prime}}=\phi_{x}+y^{\prime} \phi_{y}+z^{\prime} \phi_{x}-\phi^{2} .
$$

This set of equations for the determination of $y^{\prime}, z^{\prime}$ is of a familiar type, namely, that arising in the determination of the fixed points of a collineation, and is easily shown to admit three solutions. $\dagger$ Hence

Theorem 3. The curves defined by equations of the form (7) are such that through each point there pass three with hyperosculating circles at that point.

Since the form (7) is characterized by property $A$, it follows that the existence of three hyperosculating circles in each bundle is a consequence of property $A$.

* The radius of curvature for the curves (7) is determined by

$$
\frac{1}{r^{2}}=\phi^{2}+\psi^{2}+x^{2}-\frac{\left(\phi+y^{\prime} \psi+z^{\prime} x\right)^{2}}{1+y^{\prime 2}+z^{\prime 2}} \text {. }
$$

t Of course in special cases some of these may coincide, or the number of solutions may become infinite. The theorem stated is true "in general" in 80 far as it omits these cases which are definitely assignable. 
We state two further properties, found by considering the conditions $\left(10^{\prime}\right)$ and (11') separately.

The tangents to those curves of a system (7) which pass through a given point and there have an hyperosculating plane form a quadric cone. This cone passes through the special direction (4).

The tangents to those curves which have an osculating helix at the given point form a cubic cone. This cone passes through the special direction (4) and through the minimal directions in the plane normal to that direction.

These properties hold for natural families since they hold for all systems with property $A$. By comparing (7) with (2), we see that the functions $\phi, \psi, \chi$ in the case of a natural family are

$$
\phi=L_{x}, \quad \psi=L_{y}, \quad \chi=L_{x} ;
$$

and hence are connected by the relations

$$
\psi_{z}-x_{y}=0, \quad x_{x}-\phi_{s}=0, \quad \phi_{y}-\psi_{x}=0 .
$$

We now inquire what is the effect of these relations on the directions of the hyperosculating circles. Introducing, for symmetry,

$$
X: Y: Z=1: y^{\prime}: z^{\prime},
$$

we may write our equations (12) in the homogeneous form

$$
\begin{array}{rrr}
\chi_{y} Y^{2}-\psi_{x} Z^{2}+\left(\chi_{x}-\psi_{y}\right) Y Z & +\chi_{x} X Y-\psi_{x} X Z=0, \\
-\chi_{x} X^{2} & +\phi_{x} Z^{2} & +\phi_{y} Y Z-\chi_{y} X Y+\left(\phi_{x}-\phi^{2}-\chi_{x}\right) X Z=0, \\
\psi_{x} X^{2}-\phi_{y} Y^{2} & -\phi_{x} Y Z-\left(\phi_{x}-\phi^{2}-\psi_{y}\right) X Y+\psi_{x} X Z=0 .
\end{array}
$$

In virtue of (14), each of the quadric cones (16) is seen to be of the rectangular type.* Hence the three generators common to the cones must be mutually orthogonal. This gives

Theorem 4. In the case of any natural family the three hyperosculating circles which exist in any bundle are mutually orthogonal.

We refer to this property as property $B$.

The relations (14) are seen to be necessary as well as sufficient for the orthogonality in question. Hence property $B$ is the equivalent of (14), and serves to single out the natural families from the more general class defined by equations of form (7). The latter form was characterized by property $A$; hence we have our fundamental

Theorem 5. A system of $\infty^{4}$ curves, one for each direction at each point of space, will constitute a natural family when, and only when, it possesses prop-

* The condition for such a cone is that the sum of the coefficiente of $X^{2}, Y^{2}$, and $Z^{2}$ shall vanish. 
erties $A$ and $B$; that is, the osculating circles at any given point must form a bundle, and the three hyperosculating circles contained in such a bundle must be mutually orthogonal.

\section{§ 3. Reciprocal Systems in General.}

We have seen that the most general system of curves with property $A$ is represented by equations of the form

$$
y^{\prime \prime}=\left(\psi-y^{\prime} \phi\right)\left(1+y^{\prime 2}+z^{\prime 2}\right), \quad z^{\prime \prime}=\left(x-z^{\prime} \phi\right)\left(1+y^{\prime 2}+z^{\prime 2}\right),
$$

where $\phi, \psi, \chi$ are any functions of $x, y, z$. Such a system $S$ induces a definite point correspondence: the osculating circles at a given point $p$ pass through a second common point $P$. From the formulas given it is easily found that the coördinates of $\boldsymbol{P}$ are

$$
X=x+\frac{2 \phi}{\phi^{2}+\psi^{2}+\chi^{2}}, \quad Y=y+\frac{2 \psi}{\phi^{2}+\psi^{2}+\chi^{2}}, \quad Z=z+\frac{2 \chi}{\phi^{2}+\psi^{2}+\chi^{2}},
$$

where $x, y, z$ are of course the coördinates of $p$. This point transformation, from $p$ to $P$, we denote by $T$.

It is easily seen that $T$ is entirely general. To an arbitrary point transformation (which may even degenerate but must not be merely identity)

$$
X=X(x, y, z), \quad Y=Y(x, y, z), \quad Z=Z(x, y, z)
$$

corresponds in fact a definite system (7), namely, that system for which

$$
\phi, \psi, \chi=\frac{2(X-x), 2(Y-y), 2(Z-z)}{\Sigma(X-x)^{2}} .
$$

We assume in the remainder of the paper that the jacobian $J$ of (17) does not vanish identically, so that we may consider the inverse transformation $T^{-1}$. To this corresponds a definite system $S^{\prime}$, which may be termed the reciprocal of the given system $S$.

THeOREM 6. For any system $S$ with property $A$, that is any system (7), there is a definite reciprocal system $S^{\prime}$ of that type (provided, of course, $J$ does not vanish). The osculating circles of those curves of the system $S$ which pass through $a$ point $p$ are at the corresponding point $P$ the osculating circles of the curves of the system $S^{\prime}$ passing through $P$.

Consider the bundle of circles determined by two corresponding points $p$ and $P$. These circles osculate the $S$ curves at $p$ and the $S^{\prime}$ curves at $P$. We know that three of these circles have hyper-contact with $S$ curves. There must also be three which have hyper-contact with $S^{\prime}$ curves. We now prove that these wo triples of circles actually coincide. 
We take the point $p$ as origin and select our axes and unit of length so that, at that point, $\phi=2, \psi=0, x=0$. Then from (17) the point $P$ is $X=1$, $Y=0, Z=0$. The directions of the hyperosculating circles at $p$ are given by equations (12), which now take the form

$$
\frac{\chi_{x}+y^{\prime} \chi_{y}+z^{\prime} \chi_{z}}{z^{\prime}}=\frac{\psi_{x}+y^{\prime} \psi_{y}+z^{\prime} \psi_{x}}{y^{\prime}}=\phi_{x}+y^{\prime} \phi_{y}+z^{\prime} \phi_{x}-4
$$

From (19), we find the values, at the origin, of the partial derivatives here involved, namely,

$$
\begin{array}{lll}
\phi_{x}=2-2 X_{x}, & \phi_{y}=-2 X_{y}, & \phi_{s}=-2 X_{s}, \\
\psi_{x}=2 Y_{x}, & \psi_{y}=-2+2 Y_{y}, & \psi_{s}=2 Y_{s}, \\
\chi_{x}=2 Z_{x}, & \chi_{y}=2 Z_{y}, & \chi_{x}=-2+2 Z_{s} .
\end{array}
$$

Hence (20) may be written

$$
\text { (22) } \frac{Z_{x}+y^{\prime} Z_{y}+z^{\prime}\left(Z_{z}-1\right)}{z^{\prime}}=\frac{Y_{x}+y^{\prime}\left(Y_{y}-1\right)+z^{\prime} Y_{z}}{y^{\prime}}=-\left\{\left(X_{x}-1\right)+y^{\prime} X_{y}+z^{\prime} X_{z}\right\}-2 \text {, }
$$

which may be reduced to the form

$$
-\left(a_{1}+a_{2} y^{\prime}+a_{3} z^{\prime}\right)=\frac{b_{1}+b_{2} y^{\prime}+b_{3} z^{\prime}}{y^{\prime}}=\frac{c_{1}+c_{2} y^{\prime}+c_{3} z^{\prime}}{z^{\prime}},
$$

where the elements of the matrix

$$
\left|\begin{array}{lll}
a_{1} & a_{2} & a_{3} \\
b_{1} & b_{2} & b_{3} \\
c_{1} & c_{2} & c_{3}
\end{array}\right|
$$

stand for the elements of the jacobian

$$
J \equiv\left|\begin{array}{ccc}
X_{x} & X_{y} & X_{x} \\
Y_{x} & Y_{y} & Y_{x} \\
Z_{x} & Z_{y} & Z_{z}
\end{array}\right|
$$

In the reciprocal system $S^{\prime}$ we nust consider $x, y, z$ as functions of $X, Y$, $Z$. Hence (25) is replaced by

$$
\left|\begin{array}{lll}
x_{X} & x_{Y} & x_{Z} \\
y_{X} & y_{Y} & y_{Z} \\
z_{X} & z_{Y} & z_{Z}
\end{array}\right| \equiv \frac{1}{J}\left|\begin{array}{ccc}
A_{1} & B_{1} & C_{1} \\
A_{2} & B_{2} & C_{2} \\
A_{3} & B_{3} & C_{3}
\end{array}\right|,
$$

where $A_{1}$ for example is the minor of $a_{1}$ in (24). (The equality refers to the nine elements individually, not merely to the determinants.) In the system $S^{\prime}$ 
the directions of hyperosculation at $P$ are accordingly given by

$$
-\left(A_{1}+B_{1} Y^{\prime}+C_{1} Z^{\prime}\right)=\frac{A_{2}+B_{2} Y^{\prime}+C_{2} Z^{\prime}}{Y^{\prime}}=\frac{A_{3}+B_{3} Y^{\prime}+C_{3} Z^{\prime}}{Z^{\prime}}
$$

We wish to prove that the circles of the bundle which are determined by the three directions (23) at $p$ coincide with the circles determined by the directions (27) at $P$ Since the line from $p$ to $P$ has been taken as the axis of abscissas, the first triple of circles will pass through point $P$ in directions determined by the equations

$$
-a_{1}+a_{2} Y^{\prime}+a_{3} Z^{\prime}=\frac{-b_{1}+b_{2} Y^{\prime}+b_{3} Z^{\prime}}{Y^{\prime}}=\frac{-c_{1}+c_{2} Y^{\prime}+c_{3} Z^{\prime}}{Z^{\prime}},
$$

which are found from (23) by simply replacing $y^{\prime}$ by $-Y^{\prime}$ and $z^{\prime}$ by $-Z^{\prime}$. It is easy to verify directly that (27) and (28) give the same three solutions; the calculation may be avoided, however, by observing that the equations are precisely of the form arising in the determination of the fixed points of a given collineation and of the inverse collineation, and such collineations of course have the same fixed points. We have then

Theorem 7. Consider any system $S$ with property $A$ and the reciprocal system $S^{\prime}$ defined above. Then the three hyperosculating circles at a given point $p$ for the $S$ curves are at the same time the hyperosculating circles for the $S^{\prime}$ curves at the corresponding point $P$.

\section{§4. Reciprocity of Natural Families.}

Let us apply this result to the case where $S$ is a natural family. The hyperosculating circles at $p$ are then, according to property $B$, mutually orthogonal; the angles at the second point $P$ are obviously the same as at $p$; hence the hyperosculating circles at $P$ are mutually orthogonal, that is, the system $S^{\prime}$ will also possess property $B$. This gives our second fundamental result :

THEOREM 8. The reciprocal of a natural family is also a natural family.

Thus for each variation problem of the form

$$
\int F(x, y, z) d s=\text { minimum, }
$$

assuming that $J$ does not vanish identically, there is a second definitely related problem of the same form

$$
\int \bar{F}(x, y, z) d s=\text { minimum. }
$$

The problems may be termed reciprocal since the systems of extremals are reciprocal in the sense defined above.* We now prove

\footnotetext{
* It may happen in certain cases that the two systems coincide. Such cases arise when the transformation (17): is involutorial.
} 
Theorem 9. Given the function $F$, the related function $\bar{F}$ may be determined by eliminations and differentiations.

Letting $L$ and $\bar{L}$ denote $\log F$ and $\log \bar{F}$ respectively, we find

$$
\bar{L}=2 \int \frac{(\alpha-x) d x+(\beta-y) d y+(\gamma-z) d z}{(\alpha-x)^{2}+(\beta-y)^{2}+(\gamma-z)^{2}} ;
$$

where $\alpha, \beta, \gamma$ denote the functions inverse to the functions

$$
X=x+\frac{2 L_{x}}{L_{x}^{2}+L_{y}^{2}+L_{z}^{2}}, \quad Y=y+\frac{2 L_{y}}{L_{x}^{2}+L_{y}^{2}+L_{z}^{2}}, \quad Z=z+\frac{2 L_{z}}{L_{x}^{2}+L_{y}^{2}+L_{z}^{2}}
$$

so that

$$
x=\alpha(X, Y, Z), \quad y=\beta(X, Y, Z), \quad z=\gamma(X, Y, Z) .
$$

The differential which appears in the determination of $\bar{L}$ will necessarily be exact in virtue of the reciprocity theorem proved above.

By actual integration we find

$$
\bar{L}(X, Y, Z)=\log \left(L_{x}^{2}+L_{y}^{2}+L_{z}^{2}\right)-L .
$$

In the right hand member, the variables $x, y, z$, after the differentiations, are to be replaced by the values given in $\left(32^{\prime}\right)$. The function $\bar{L}(x, y, z)$ may be obtained from the function $\log \left(L_{x}^{2}+L_{y}^{2}+L_{x}^{2}\right)-L$ by substituting for $x, y, z$, the functions $\alpha(x, y, z), \beta(x, y, z), \gamma(x, y, z)$, respectively.

\section{§ 5. The Transformations $T$ and $T_{1}$. Darboux Deformations.}

The point transformation $T$ connected with the general $S$ system (7), by means of which the point $p$ corresponds to the second vertex $\boldsymbol{P}$ of the bundle of osculating circles, according to the formulas

$$
X=x+\frac{2 \phi}{\phi^{2}+\psi^{2}+\chi^{2}}, \text { etc., }
$$

is entirely general. If however the $S$ system is a natural family, then $\boldsymbol{T}$ must have some peculiarity. What is the character of the transformation $T$ in the case of a natural family? That is, what is the effect on $T$ of the relations

$$
\psi_{x}-\chi_{y}=0, \quad \chi_{x}-\phi_{x}=0, \quad \phi_{y}-\psi_{x}=0 ?
$$

To answer this we consider first another transformation

$$
X_{1}=x+\phi, \quad Y_{1}=y+\psi, \quad Z_{1}=z+x .
$$

This associates with the point $p$ a point $P_{1}$ which is easily shown to be the pole, with respect to a unit sphere about $p$ as center, of the plane bisecting perpendicularly the line from $p$ to $P$, that is, the plane containing the centers of 
curvature. Otherwise stated, $p, P$, and $P_{1}$ are collinear and

$$
\overline{p P} \cdot \overline{p P}{ }_{1}=2 \text {. }
$$

Thus each of the transformations $T$ and $T_{1}$ uniquely determines the other. Hence any property of $T_{1}$ is virtually a property of $T$. We wish to prove

TheOREM 10. For any natural family the associated transformation $T_{1}$ is of the Darboux type (defined below). Conversely, if $T_{1}$ is of this type, the corresponding $S$ system will be natural.

The category of transformations here described as of the DARBoux type were met with by Darboux in the study of deformations of space.* For an arbitrafy space deformation

$$
x_{1}=\Phi(x, y, z), \quad y_{1}=\Psi(x, y, z), \quad z_{1}=\chi(x, y, z),
$$

there exist at each point three directions which are converted into parallel directions. This triplet will, at every point of space, be tri-rectangular when, and only when,

$$
\Psi_{s}-\chi_{y}=0, \quad \chi_{x}-\Phi_{z}=0, \quad \Phi_{y}-\Psi_{x}=0 .
$$

This is the criterion of a DARBoux transformation.

Applying the test to $T_{1}$ we readily verify theorem 10 . The property here proved is seen to be an equivalent of property $B$. Thus natural systems may be characterized completely, without introducing hyperosculating circles, as follows : $\dagger$

The osculating circles at a given point must form a bundle (property $A$ ); and the associated trausformation $T_{1}$, which is then induced, must be such that the three directions at each point which are converted into parallel directions are tri-rectangular (property $D$ ).

It is obvious that the inverse of any DaRBoux transformation will also be of that type. $\neq$ The reciprocity theory for natural systems is not however contained in this statement. In connection with any transformation $T$ we consider, in addition to the inverse transformation $t=T^{-1}$, two other transformations $T_{1}$ and $t_{1}$. If $T$ converts point $p$ into point $P$, then $T_{1}$ converts $p$ into $P_{1}$ and $t_{1}$ converts $P$ into $p_{1}$, where $P_{1}$ and $p_{1}$ are two points on the line $p P$ so situated that

$$
\overline{p P} \cdot \bar{p} P_{1}=2, \quad \overline{P p} \cdot \overline{P p_{1}}=2 \text {. }
$$

* Proceedinga of the London Mathematioal Society, 1900.

† The statement $D$ is preferable to statement $B$ in so far as the caloulations to whioh it leads are simpler and more easily extended to higher dimensions. But $B$ is on the whole better since it renders the reciprocity relation intuitive. The three special directions described in $D$ are not in general the same as those appearing in $B$.

$¥$ The totality of DABBoux transformations do not form a group with respect to the ordinary multiplicative combination. They do, however, form a group with respect to addition defined as follows: if one transformation converts $P$ into $P_{1}$, and a second converts $P$ into $P_{2}$, then the oum converts $P$ into $P_{3}$, such that the vector $P P_{3}$ equals the sum of the vectors $P P_{1}$ and $P P_{2}$. Analytically, $x_{3}=x_{1}+x_{2}-x$, eto. 
Usually, of these four transformations, any one of which determines the other three, none is of the Darboux type. The reciprocity theory in $\S 4$ amounts to the following

Theorem 11. When $T_{1}$ is of the Darboux type so also is $t_{1}$.

For this means, according to theorem 10 , that whenever $S$ is a natural system so also is the reciprocal system $S^{\prime \prime}$.

It may be shown that the only case in which the transformation $T$ connected with a natural system is of the DARBoux type is that in which the function $L$ satisfies an equation of the form

$$
L_{x}^{2}+L_{y}^{2}+L_{x}^{2}=\text { function of } L .
$$

This means that the surfaces $L=$ constant must be parallel. Hence in the dynamical interpretation the lines of force must be straight. We have thus a characterization of the rectilinear conservative fields of force.

\section{§6. Velocity Srstems.}

The most general system with property $A$ is represented by differential equations of the form

$$
y^{\prime \prime}=\left(\psi-y^{\prime} \phi\right)\left(1+y^{\prime 2}+z^{\prime 2}\right), \quad z^{\prime \prime}=\left(\chi-z^{\prime} \phi\right)\left(1+y^{\prime 2}+z^{\prime 2}\right),
$$

and thus involves three arbitrary functions. Only in the case where these functions are the partial derivatives of the same function is the system a natural one. We now point out a dynamical problem that leads to the general type (7).

Consider a particle (of unit mass) moving in any field of force, the components of the force being $\phi, \psi, \chi$. The equations of motion are then

$$
\ddot{x}=\phi(x, y, z), \quad \ddot{y}=\psi(x, y, z), \quad \ddot{z}=x(x, y, z) .
$$

If the initial position and the initial velocity are given the motion is determined. If only the initial position and direction of motion are given, the osculating plane will be determined but the radius of curvature $r$ will depend for its value on the initial speed $v$. Hence, in addition to the usual formula

$$
v^{2}=\dot{x}^{2}+\dot{y}^{2}+\dot{z}^{2},
$$

there must be a formula expressing $v^{2}$ in terms of $x, y, z, y^{\prime}, z^{\prime}, r$. This is furnished by the familiar equation

$$
v^{2}=r N
$$

where $N$ denotes the (principal) normal component of the force, so that

$$
N^{2}=\phi^{2}+\psi^{2}+\chi^{2}-\frac{\left(\phi+y^{\prime} \psi+z^{\prime} \chi\right)^{2}}{1+y^{\prime 2}+z^{\prime 2}}
$$

The result may be written in the two forms

$$
v^{2}=\frac{\left(\psi-y^{\prime} \phi\right)\left(1+y^{\prime 2}+z^{\prime 2}\right)}{y^{\prime \prime}}=\frac{\left(\chi-z^{\prime} \phi\right)\left(1+y^{\prime 2}+z^{\prime 2}\right)}{z^{\prime \prime}} .
$$


In the actual trajectory $v$ varies from point to point. If now we replace $v^{2}$ in this result by some constant, say $1 / c$, the resulting equations may be written

$$
\begin{aligned}
& y^{\prime \prime}=c\left(\chi-y^{\prime} \phi\right)\left(1+y^{\prime 2}+z^{\prime 2}\right), \\
& z^{\prime \prime}=c\left(\chi-z^{\prime} \phi\right)\left(1+y^{\prime 2}+z^{\prime 2}\right) .
\end{aligned}
$$

The curves satisfying these differential equations - they are not in general trajectories - we define as velocity curves. In a given field of force there are $\infty^{5}$ trajectories and $\infty^{5}$ velocity curves.* If $c$ is given we have $\infty^{4}$ velocity curves. In particular if $c$ (and hence $v$ ) is taken to be unity, our equations become precisely (7).

TheOREM 12. Any system of $\infty^{4}$ curves possessing property $A$, that is, any system (7), may be regarded as the totality of velocity curves corresponding to unit velocity in some (uniquely defined) field of force.

Only when the field is conservative do the velocity systems for each value of $v$ (or $c$ ) become natural systems. The trajectories also are in this case made up of $\infty^{1}$ natural families, one for each value of $h$ the energy constant; but the two sets of natural families are distinct. The determination of a velocity system in one conservative field is equivalent to the determination of a trajectory system in another conservative field, and vice versa. We find in fact the following

TheOREM 13. If two conservative fields with work functions $W_{1}$ and $W_{2}$ satisfy the relation $\uparrow$

$$
W_{2}=a e^{\frac{2 W_{1}}{\sigma_{b}^{2}}}-h
$$

then the $\infty 4$ velocity curves for the speed $v_{0}$ in the first field coincide with the $\infty^{4}$ trajectories for the constant of energy $h$ in the second field. $\neq$

Halphen (Comptes rendus, vol. 84) showed that the only forces in space for which all the $\infty^{5}$ trajectories are plane curves are the central and parallel forces. We inquire when the system of $\infty^{4}$ velocity curves is made up of plane curves, and find that the requisite condition is

$$
\Sigma\left(\psi-y^{\prime} \phi\right)\left(x_{x}+y^{\prime} \chi_{y}+z^{\prime} \chi_{z}\right) \equiv 0,
$$

where the summation refers to circular permutation of $\phi, \psi, \chi$. This leads to precisely the same set of partial differential equations that appear in HaLPHEN's problem. Hence we have

\footnotetext{
* The properties of a complete system of $\infty^{5}$ velocity ourves are analogous to, but distinot from, those of a complete system of trajectories.

+ We note that if $W_{1}$ is left unaltered and $v_{0}$ varied, $W_{2}$ takes quite distinct forms. The $\infty \infty^{1}$ velocity systems in a given field do not constitute the complete system of $\infty^{5}$ trajectories in any field whatever.

$\ddagger$ It is seen that the two fields have the same equipotential surfaces and therefore the same lines of force. (Central fields therefore correspond to central fields.)
} 
Theorem 14. The $\infty^{4}$ curves of a system of type (7), that is, of a velocity system, will be plane curves when and only when the vector field defined by $\phi, \psi, \chi$, is central or parallel.

These two corollaries are of interest :

If in a conservative field the trajectories corresponding to one value of the constant of energy $h$ are plane curves, the same will be true for all values of $h$, and the potential will vary according to some function of the distance from either a fixed point or a fixed plane.

The only cases in which the extremals connected with the variation problem

$$
\int F(x, y, z) d s=\text { minimum }
$$

are plane curves are those in which $F$ is expressible as a finction of a single argument of the type *

$$
c_{0}\left(x^{2}+y^{2}+z^{2}\right)+c_{1} x+c_{2} y+c_{3} z+c_{4} \text {. }
$$

\section{§ 7. Conformal Transformations.}

If an arbitrary point transformation is applied to space, the $\infty^{4}$ curves of a natural family will not usually be converted into curves constituting a natural family. We now prove the following

Theовем 15. The only point transformations which convert every natural family into a natural family are those belonging to the conformal group.

This may be proved by means of the differential equations of the family, but. appears more readily from the variation problem (1). If an integral of this form is to be converted into one of the same form, the transformation must convert $F d s$ into an expression of the same type, say $F_{1} d s$. Therefore $d s$ must be converted into a multiple of itself ; that is, the equation $d s=0$, defining the minimal lines, must be invariant. This is a familiar characteristic property of conformal transformations. Hence our result is established.

Natural systems have properties $A$ and $B$. It is of interest to notice that property $A$ by itself is conformally invariant. More precisely we have

Theorem 16. If a conformal transformation is applied to any system possessing property $A$, that is, any system of the type (7), the new system will also be of that type. No other point transformations leave type (7) invariant. $\dagger$

The proof of the last part of the theorem is most easily obtained by noticing that the equations of any system (7), namely,

$$
y^{\prime \prime}=\left(\psi-y^{\prime} \phi\right)
$$

$$
\left(1+y^{\prime 2}+z^{\prime 2}\right)
$$

$$
z^{\prime \prime}=\left(x-z^{\prime} \phi\right)\left(1+y^{\prime 2}+z^{2}\right),
$$

* The surfaces $F=$ constant must then be either concentria spheres or parallel planes. The result is made most concrete by the optical interpretation in which $F$ equals $v$ the index of refraction. The anthor has applied this result to determine all isotropic media in which the rays of light are ciroular.

It may be shown that, for any non-conformal transformation, there exists a unique system $\alpha$ type (7), i. e., a unique velocity system, which is converted into a velocity system. 
admit as particular integrals the solutions of the set

$$
1+y^{\prime 2}+z^{\prime 2}=0, \quad y^{\prime \prime}=0, \quad z^{\prime \prime}=0 .
$$

The latter equations obviously represent the minimal straight lines of space. No other solutions are common to all systems (7). Hence the $\infty^{3}$ minimal straight lines are the only lines (straight or curved) included in every system with property $A$. If therefore a transformation is to leave type (7) invariant, it must leave this part, common to all systems of that type, invariant. Hence no other than conformal transformations can fulfill our conditions.

The first part of the theorem may be proved synthetically.* The $\infty^{10}$ conformal transformations of space convert circles into circles, and bundles of circles into bundles of circles. Hence if the original system possesses property $A$, the same will be true of the transformed system.

If the system possesses property $B$ in addition to $A$, the same will obviously be true after conformal transformation; for hyperosculating circles will remain such and the tri-rectangular aspect will be preserved.

\section{§ 8. Two-dimenstonal Results.}

We have seen that natural systems of curves constitute a special species of the general class made up of all velocity systems. In the case of the plane, our systems contain $\infty^{2}$ curves (instead of $\infty^{4}$ as in space); the velocity type becomes

$$
y^{\prime \prime}=\left(\Psi-y^{\prime} \phi\right)\left(1+y^{\prime 2}\right),
$$

involving two arbitrary functions $\phi(x, y), \psi(x, y)$; and the natural type is

$$
y^{\prime \prime}=\left(L_{y}-y^{\prime} L_{x}\right)\left(1+y^{2}\right) \text {, }
$$

corresponding to the restriction

$$
\phi_{y}-\psi_{x}=0
$$

Another noteworthy case arises in the theory of isogonal trajectories. If for a given system of $\infty^{1}$ curves all the isogonals are constructed, the resulting system of $\infty^{2}$ curves has an equation of the form

$$
y^{\prime \prime}=\left(\Omega_{x}+y^{\prime} \Omega_{y}\right)\left(1+y^{\prime 2}\right) \text {. }
$$

This corresponds to a different restriction, namely,

$$
\phi_{x}+\psi_{y}=0 \text {. }
$$

Theorem 17. Included in the velocity type, in the case of troo dimensions,

* This part of the reasoning would be insufficient in the case of two dimensions, since it would be valid only for the six-parameter group of MöBIUs ciroular transformations. Direot caloulation, however, establishes the truth of the result for the infinite conformal group of the plane. Ct. American Journal of Mathematios, vol. 27 (1906), p. 213. 
are two noteworthy species: the natural and the isogonal. The former arises when the vector field $\phi, \psi$ is lamellar, the latter when the field is solenoidal.*

For each velocity system $S$ there is a reciprocal system $S^{\prime}$. Our discussion shows that when $S$ is natural so also is $S^{\prime}$, and SchefFer's work shows that when $S$ is isogonal $S^{\prime}$ is also. However, systems $S$ for which

$$
J \equiv \text { jacobian }\left(x+\frac{2 \phi}{\phi^{2}+\psi^{2}}, y+\frac{2 \psi}{\phi^{2}+\psi^{2}}\right)
$$

vanishes identically do not give rise to a reciprocal system. $\dagger$

The only systems which are common to the two species described are those which satisfy both restrictions

$$
\phi_{y}-\psi_{x}=0, \quad \phi_{x}+\psi_{y}^{j}=0 .
$$

The function $L$ and also the function $\Omega$ will then be harmonic. The type arising will then consist of velocity systems in Laplacian fields, or, what is equivalent, of the isogonals of an isothermal system.f

It follows that if $S$ is composed of the isogonals of an isothermal system the same will be true of the reciprocal system $S^{\prime}$.

Every natural system in the plane may be regarded as the conformal representation of the geodesics of some surface. From this point of view we may state

Theorem 18. A system of $\infty^{2}$ plane curves, one through each point in each direction, may be regarded as the conformal representation of the system of geodesics on some surface when and only when $(A)$ the circles of curvature constructed at a given point form a pencil and $(B)$ the two hyperosculating circles existing in such a pencil are orthogonal.

The reciprocity theorem takes the following form: With every conformal representation of any surface $\sigma$ upon a plane there is associated a conformal representation of another surface $\sigma^{\prime}$ upon the plane, defined by the fact that the two systems in the plane which depict the two systems of geodesics are reciprocal systems $S$ and $S^{\prime}$.

If the first conformal representation is given, $S$ and hence $S^{\prime}$ is determined;

\footnotetext{
* Condition (46) means that the ourl vanishes, while (47') means that the divergence vanishes.

† The existence of such exceptional cases is apparently not brought out in SCHEFFER's theory. Reciprocal isogonal systems may be regarded, from a certain point of view, as images of surfaces conjugate with respect to a null system. The exceptional systems then appear as images of developable surfaces.

$\ddagger$ Cf. the anthor's note, Isothermal systems in dynamics, Bulletin of the A merioan Mathematical Society, vol. 14 (1908), pp. 169-172. The Laplacian fields in the plane (i. e., those with a harmonic potential) are distinguished from all others by the fact that each of the $\infty^{1}$ syetems of velocity curves is a linear two-parameter system.
} 
the corresponding surface $\sigma^{\prime}$ is then fixed except for isometric deformation and homothetic transformation. *

The curvature transformation $\Gamma$.

Any conformal transformation of the plane converts the general type of velocity system

$$
y^{\prime \prime}=\left(\psi-y^{\prime} \phi\right)\left(1+y^{\prime 2}\right)
$$

into itself ; $\uparrow$ furthermore, each species, the natural (or geodesic) and the isogonal, is separately invariant.

We now show that the following transformation

$$
x_{1}=x, \quad y_{1}=y, \quad y_{1}^{\prime}=-\frac{1}{y^{\prime}}, \quad y_{1}^{\prime \prime}=-\frac{y^{\prime \prime}}{y^{\prime 3}}
$$

interchanges the troo species. Applying it in fact to the general type (45), we find that the new differential equation is of the same form, with $\phi$ and $\psi$ replaced respectively by $\phi_{1}$ and $\psi_{1}$, where

$$
\phi_{1}=-\psi, \quad \psi_{1}=\phi .
$$

Hence if the original field $\phi, \psi$ is solenoidal the new field $\phi_{1}, \psi_{1}$ will be conservative, and vice versa. We state the result as

Theorem 19. The isogonal system

$$
y^{\prime \prime}=\left(\Omega_{x}+y^{\prime} \Omega_{y}\right)\left(1+y^{\prime 2}\right),
$$

which consists of all the isogonals of the simple system

$$
y^{\prime}=\tan ^{-1} \Omega,
$$

is converted by $\Gamma$ into the natural system

$$
y^{\prime \prime}=\left(\Omega_{y}-y^{\prime} \Omega_{x}\right)\left(1+y^{\prime 2}\right),
$$

which may be obtained as the conformal representation of the geodesics of the surface whose squared element of length is

$$
d s^{2}=e^{2 \Omega}\left(d x^{2}+d y^{2}\right) .
$$

The transformation $\Gamma$ is obviously not a contact transformation. It may be defined as the operation by means of which any curvature element $\left(x, y, y^{\prime}, y^{\prime \prime}\right)$ is converted into another curvature element $\left(x, y,-1 / y^{\prime},-y^{\prime \prime} / y^{\prime 3}\right)$ obtained from the first by rotating it about its own point $(x, y)$ through a right angle, its curvature being preserved. $\neq$

* If $\sigma$ is a developable surface the same is true of $\sigma^{\prime}$. It is possible that there are other cases in which the two eurfaces are isometrically equivalent.

†Cf. American Journal of Mathematics, vol. 28 (1906), p. 213.

$\ddagger$ The operation $\Gamma^{2}$ converts each curvature element into its opposite $\left(x, y, y^{\prime},-y^{\prime \prime}\right)$. This process leaves each of our two species invariant. 
With each natural system there is associated, by means of $\Gamma$, a definite isogonal system, and vice versa. Hence every property of the one species gives indirectly a property of the other. Thus for an isogonal system, while it is not true that the two hyperosculating circles in each pencil are orthogonal, this will be true for the associated system derived by means of $\Gamma$.

We are now in position to complete the theory of isogonals. The CesaroScheFfers theorem asserts that for such a system the circles of curvature at a given point $p$ pass through a second common point $P$. Construct the point $P_{1}$ on the segment $p P$ such that $p P \cdot p P_{1}=2$, and also the point $P_{2}$ obtained by rotating the segment $p P_{1}$ about $p$ through a right angle. The transformation $T_{2}$ which converts every point $p$ into the point $P_{2}$ determined in this way will then be of the Darboux type,* that is, will be such that the two lineal elements at each point which are converted into parallel elements are perpendicular to each other.

Theorem 20. Hence a system of $\infty^{2}$ plane curves will be a system of isogonals when, and only when, in addition to the CEsARo-SchefFers property, it satisfies the requirement that the induced transformation $T_{2}$ (defined above) is of the Darboux type.

Thus if a transformation $T$ is given the velocity system thereby determined will be a natural or an isogonal system in those cases where the related $T_{1}$ or $T_{2}$ transformation is of the DARBoux type.

To what extent may the members of a system of specified type (velocity, natural, or isogonal) be taken arbitrarily? If a net of curves is given, $i . e .$, two curves through each point of the plane, then a velocity system may be constructed to which that net belongs. A natural system, like an isogonal system, is determined by any $\infty^{1}$ curves, one through each point of the plane (or the region considered), together with an arbitrary transversal curve (not tangent to any of the $\infty^{\prime}$ curves). This result, which is fairly intuitive for the isogonal case, may be proved without difficulty by means of the $\mathrm{CAUCH}_{\mathrm{Y}}$ existence theorem for partial differential equations.

Columbia University.

* The transformation $T_{1}$ associated with an isogonal system will be of the DARBOUX type only in the isothermal case. It may be shown that, for any isogonal system, $T$, has the following characteristio property : at any point the two lineal elements each of which is converted into a perpendicular element are perpendicular to each other. 